\title{
Detección de albinismo en la regeneración de Persea lingue y Cryptocarya alba procedente del cerro Cayumanque, Región del Biobío, Chile
}

\section{Albinism detection among Persea lingue and Cryptocarya alba regeneration, from Cayumanque Mountain, Biobío Region, Chile}

\author{
Carolina Álvarez ${ }^{1 *}$, Manuel Acevedo ${ }^{1}$, Marta González $^{1}$, Eduardo Cartes $^{1}$ \& Jan Bannister ${ }^{2}$ \\ ${ }^{1}$ Instituto Forestal sede Biobío, Camino a Coronel km 7,5, San Pedro de la Paz, Concepción, Chile. \\ ${ }^{2}$ Instituto Forestal sede Los Ríos, Guarategua Lepe s/n, Nercón, Chiloé, Chile. \\ *calvarez@infor.cl
}

\begin{abstract}
As part of the ecological restoration of the Cerro Cayumanque, affected by a fire in 2012, seeds of native species were collected for plant production in nursery and posterior introduction. Here, albino seedlings of Cryptocarya alba and Persea lingue were detected. This is the first report of detection of a lethal mutation such as albinism within the regeneration of Chilean native species. In C. alba $5 \%$ of albinism and $0,1 \%$ in P. lingue were detected among seedlings.
\end{abstract}

Actualmente, la disminución de la biodiversidad es una de las principales preocupaciones a nivel global (Barnosky et al. 2011). Un factor que afecta gravemente la biodiversidad de las especies es la fragmentación y el deterioro del hábitat, donde las poblaciones se hacen más pequeñas y aisladas unas de otras, disminuyendo el número de individuos en la población y el flujo de genes (Ellstrand \& Elam 1993, Dudash \& Fenster 2000). En Chile, los ecosistemas mediterráneos han sido los más afectados por la fragmentación del hábitat. Estos sistemas cubren alrededor de un $16 \%$ del territorio continental, y son el hábitat de cerca de un $50 \%$ de las especies de plantas y vertebrados endémicos (Arroyo et al. 2004). Un claro ejemplo de fragmentación y destrucción del hábitat es el cerro Cayumanque, un área propuesta como sitio prioritario para la conservación de la biodiversidad (Bannister et al. 2012, Ibarra-Vidal et al. 1998), que fue afectado por el incendio denominado Pichiqueime en la provincia de Nuble, Región del Biobío, afectando una superficie de 26.640 ha en total, devastando 1.789 ha de bosque nativo.

En el marco del "Programa Restauración Ecosistema Cayumanque" del Ministerio del Medio Ambiente, el Centro Tecnológico de la Planta Forestal del Instituto Forestal produjo durante la temporada 2015-2016 plantas de especies nativas a partir de semillas colectadas en el cerro Cayumanque. El principal objetivo de este programa es la propagación de plantas de especies nativas afectadas por el incendio, mediante semillas colectadas de la misma procedencia. Sin embargo, en el vivero durante el proceso de germinación de las especies Cryptocarya alba (Mol.)
Looser y Persea lingue (Ruiz \& Pav.) Nees, se detectó la presencia de plantas albinas.

El reporte de este tipo de mutaciones letales es de gran importancia, ya que en poblaciones pequeñas y aisladas la cruza entre parientes se vuelve cada vez más común, disminuyendo la variabilidad genética a través del aumento del nivel de homocigocidad (Ellstrand \& Elam 1993, Lande 1995, Young et al. 1996). En poblaciones fragmentadas, la deriva genética puede sobrepasar la fuerza de la selección y llevar a una fijación acumulativa de alelos recesivos homocigotos que reducen la aptitud de una población (Willi $\&$ Fischer 2005, Busch 2006) y aumentan la presencia de individuos con mutaciones letales, como el albinismo. Así, la endogamia es una amenaza que puede afectar directamente la persistencia de una población, convirtiéndose en una preocupación para la restauración (Ellstrand \& Elam 1993, Vandepitte et al. 2010). Este documento tiene por objetivo reportar la aparición de albinismo en plántulas de C. alba y $P$. lingue, procedentes del cerro Cayumanque, durante el proceso de viverización.

Las semillas fueron recolectadas directamente de árboles de $C$. alba y $P$. lingue ubicados en el predio Santa Gertrudis del cerro Cayumanque (36 $\left.32^{\prime} \mathrm{S} 72^{\circ} 31^{\prime} \mathrm{O}\right)$. La limpieza, siembra de semillas y viverización de plantas se realizó en las instalaciones del vivero del Centro Tecnológico de la Planta Forestal del INFOR, en la comuna de San Pedro de la Paz, Región del Biobío.

La viverización se realizó de acuerdo a Monsalve et al. (2009), en bandejas de poliestireno expandido de 84 cavidades y con un volumen por cavidad de $130 \mathrm{~cm}^{3}$. 
Las bandejas fueron bañadas con una mezcla de látex y oxicloruro de cobre, con el fin de promover la poda química de las raíces, utilizando como sustrato corteza compostada de pino radiata calibre G-10. Las semillas fueron sembradas directamente, sin la aplicación de tratamientos pregerminativos, durante septiembre de 2015.

Para la determinación de plantas albinas se consideraron plantas de color blanco y se realizaron mediciones de eficiencia fotoquímica del PSII (Fv/Fm) en C. alba y P. lingue, para evaluar si las plantas albinas eran fotosintéticamente activas. Para esto se utilizó un fluorímetro Plant Efficiency Analizer (PEA, Hansatech Instruments, UK). Hojas de seis individuos de cada condición fueron oscurecidas por 30 min para posteriormente realizar la determinación a una intensidad de luz saturante no modulada de $2400 \mu \mathrm{mol}$ fotones $\mathrm{m}^{-2} \mathrm{~s}^{-1}$.

Las mediciones de $\mathrm{Fv} / \mathrm{Fm}$, tanto en $C$. alba como en $P$. lingue presentaron valores de cero para todos los individuos albinos medidos, indicando que este tipo de plantas no son fotosintéticamente activas. Por el contrario, plantas no albinas de ambas especies presentaron valores de $\mathrm{Fv} / \mathrm{Fm}$ de 0,795 $( \pm 0,0208)$ en $C$. alba, y $0,823( \pm 0,0215)$ en $P$. lingue. En $C$. alba se observó un $5 \%$ de albinismo, lo que corresponde a 1.487 plantas albinas de 28.980 plantas producidas (Fig. 1a). Para el caso de $P$. lingue se observó solamente un $0,1 \%$ de albinismo, correspondiente a 8 albinas de un total de 6.000 plantas producidas (Fig. 1b). Este fenómeno no se observó en otras especies de la producción de plantas procedentes del cerro Cayumanque, lo que fue evaluado de manera visual (Laurelia sempervirens (Ruiz \& Pav.) Baill, Aextoxicon punctatum (Ruiz \& Pav.), Lomatia dentata (Ruiz \& Pav.), Quillaja saponaria (Molina), Nothofagus obliqua (Mirb). y Luma apiculata (DC.) Burret). Los individuos albinos fueron evidenciados desde el momento de su emergencia y murieron alrededor de dos meses posteriores a ésta.

La detección de mutaciones letales que se expresan tempranamente en el ciclo de vida es extremadamente rara, ya que dejan una mayor cantidad de individuos heterocigotos para muestrear en las semillas o plántulas sobrevivientes (Savolainen \& Hendrick 1995). Por otra parte, si bien el albinismo puede ser resultado de una mutación natural, es generalmente un fenómeno asociado a cultivos creados a partir de híbridos o utilizando tecnologías de cultivo in vitro (Kumari et al. 2009), por lo que las investigaciones se centran principalmente en este último caso.

En relación a poblaciones naturales, en Dactylis glomerata L., se ha reportado la aparición de plántulas albinas entre un 12 y $15 \%$ de la progenie (Apirion \& Zohary 1961), mientras que en Picea pungens Engelm. se ha detectado en un $6 \%$, aproximadamente (Cram 1963). Estos resultados son similares a los reportados por Willis (1992) en Erythranthe guttata Nesom autopolinizados.

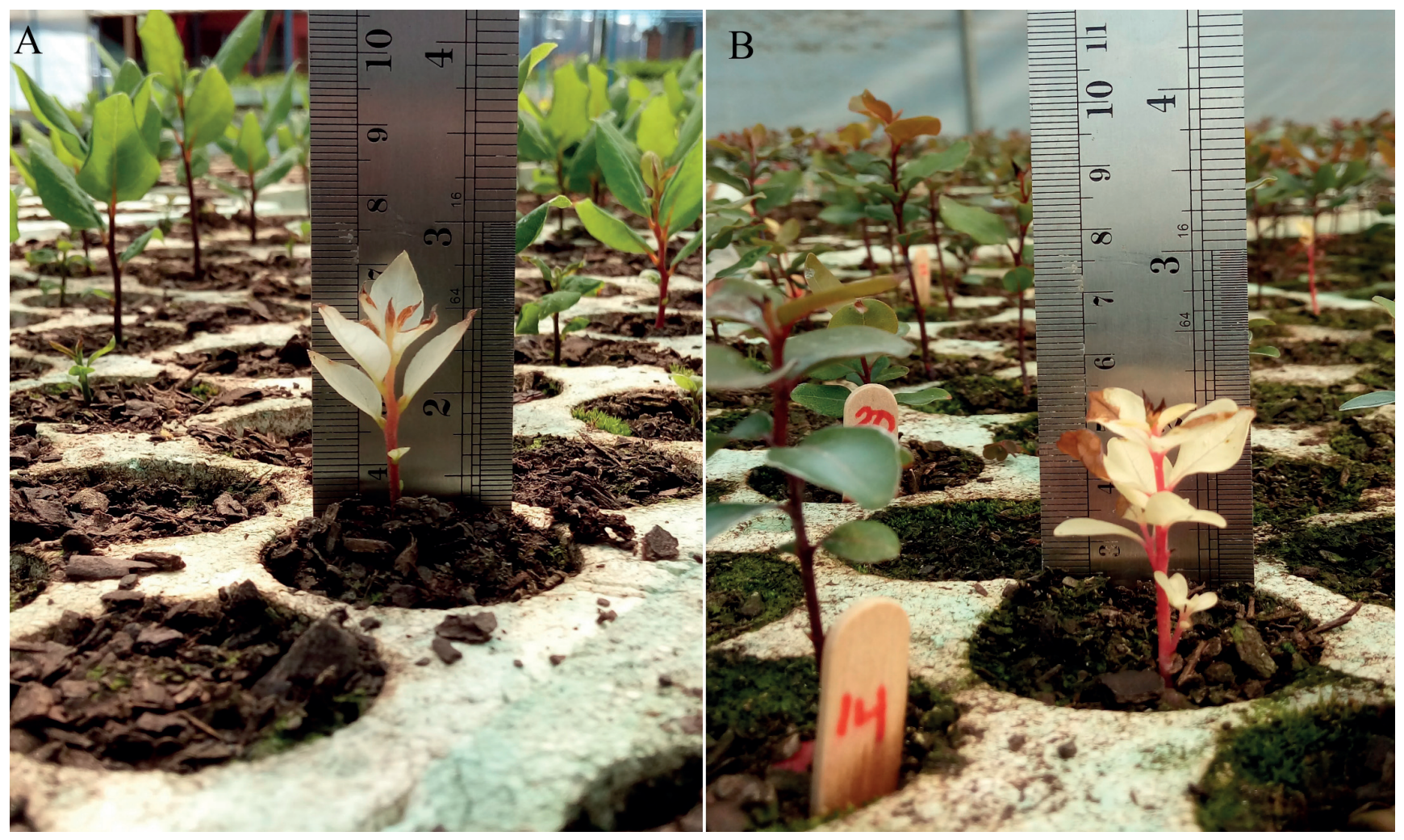

Figura 1. Plántulas albinas de Persea lingue (a) y Cryptocarya alba (b) procedentes del cerro Cayumanque. / Albino seedlings of Persea lingue (a) and Cryptocarya alba (b) from Cayumanque Mountain. 
La detección de mutaciones letales y deletéreas se asocia generalmente a disminución en la diversidad genética y aumento de tasa de endogamia en poblaciones naturales. Así, Shreekumari \& Abraham (2005), indicaron una mayor frecuencia de albinismo en plantas con endogamia en híbridos de Amorphophallus paeoniifolius (Dennst.) Nicolson, mientras que Us-Camas et al. (2017) observaron baja variabilidad genética entre fenotipos normales, albinos y variegados de Agave angustifolia Haw.

Así, la detección de mutaciones letales como el albinismo, podría ser un indicador clave de disminución de variabilidad genética en estas especies, causando disminución en la capacidad adaptativa a nuevas condiciones ambientales, acumulación de mutaciones letales y deletéreas, y disminución de las aptitudes de los individuos de una población. En consecuencia, obtener esta información es vital para la correcta implementación de los programas de restauración. Este deterioro podría indicar que la utilización de material vegetal procedente del mismo sitio a restaurar podría no ser la mejor estrategia, ya que se estarían introduciendo individuos con disminución en su desempeño, contribuyendo a la aparición de mutaciones y disminución de la aptitud de futuras generaciones.

Considerando estos antecedentes, pareciera pertinente evaluar a futuro el deterioro genético de fragmentos poblacionales que están en procesos de restauración.

\section{AGRADECIMIENTOS}

A la Secretaría Regional Ministerial del Medio Ambiente del Biobío por apoyar este trabajo el cual se inserta en el Programa Restauración Ecosistema Cayumanque.

\section{REFERENCIAS}

Arroyo, M.T.K., Marquet, P.A., Marticorena, C., Simonetti, J.A., Cavieres, L.A., SQueo, F.A., Rozzi, R. 2004. Chilean winter rainfall-Valdivian forest. En: Mittermeier, R.A., Gil, P.R., Hoffman, M., Pilgrim, J., Brooks, T., Mittermeier, C.G., Lamoreux, J., Fonseca, G.A.B. (eds.), Hotspots Revisted: Earth's Biologically Wealthiest and most Threatened Ecosystems. pp. 99-103. CEMEX, México D.F.

ApIrion, D., Zohary, D. 1961. Chlorophyll lethal in natural populations of the orchard grass (Dactylis glomerata L.). A case of balanced polymorphism in plants. Genetics 46(4): 393-399.

Bannister, J.R., Vidal, O.J., Teneb, E., Sandoval, V. 2012. Latitudinal patterns and regionalization of plant diversity along a 4,270 km gradient in continental Chile. Austral Ecology 37(4): 500-509.

Barnosky, A.D., Matzke, N., Tomiya, S., Wogan, G.O.U., Swartz,
B., Quental, T.B., Marshall, C., McGuire, J.L., Lindsey, E.L., Maguire, K.C., Mersey, B., Ferrer, E.A. 2011. Has the earth's sixth mass extinction already arrived? Nature 471(7336): 51-57.

Busch, J. 2006. Heterosis in an isolated, effectively small, and self fertilizing population of the flowering plant Leavenworthia alabamica. Evolution 60(1): 184-191.

CRAM, W.H. 1963. Albinism and natural selfing in Picea pungens. Canadian Journal of Plant Sciences 63(4): 1097-1098.

Dudash, M.R., Fenster, C.B. 2000. The role of breeding system and inbreeding depression in the maintenance of an outcrossing mating strategy in Silene virginica (Caryophyllaceae). American Journal of Botany 88(11): 1953-1959.

Ellstrand, N., Elam, D. 1993. Population genetic consequences of small population size: implications for plant conservation. Annual Review of Ecology, Evolution and Systematics 24(10): 217-242.

Ibarra-Vidal, H., Ortiz, J., Rodríguez, R., Pauchard, A., Verdugo, R., Maldonado, E. 1998. Cerro Cayumanque (Octava Región): un sitio prioritario para la conservación de la biodiversidad en Chile: Antecedentes para su protección como santuario de la naturaleza. Informe Artículos 11. Chile.

Kumari, M., Clarke, H.J., Small, I., Siddique, K.H.M. 2009. Albinism in plants: A major bottleneck in wide hybridization, androgenesis and double haploid culture. Critical Reviews in Plant Science 28: 393-409.

LANDE, R. 1995. Mutation and conservation. Conservation Biology 9(4): 782-791.

Monsalve, J., Escobar, R., Acevedo, M., Sánchez, M., Coopman, R. 2009. Efecto de la concentración de nitrógeno sobre atributos morfológicos, potencial de crecimiento radical y estatus nutricional en plantas de Eucalyptus globulus producidas a raíz cubierta. Bosque 30(2): 88-94.

Savolainen, O., Hendrick, P. 1995. Heterozygosity and fitness: no association in Scot's Pine. Genetics 140: 755-766.

Shreekumari, M.T., Abraham, K. 2005. Occurrence of albino seedlings in elephant foot yam. Journal of Root Crops 31 : 61-62.

Us-Camas, R., Castillo-Castro, E., Aguilar-Espinosa, M., Limónes-Briones, V., Rivera-Madrid, R., Robert-Díaz, M., De la PeÑa, C. 2017. Assessment of molecular and epigenetic changes in the albinism of Agave angustifolia Haw. Plant Science 263: 156-167. https://doi.org/10.1016/j. plantsci.2017.07.010

Young, A., Boyle, T., Brown, A. 1996. The population genetic consequences of habitat fragmentation for plants. Trend in Ecology and Evolution 11(10): 413-418.

Vandepitte, K., Honnay, O., Jacquemyn, H., Roldán-Ruiz, I. 2010. Effects of outcrossing in fragmented populations on the primarily selfing forest herb Geum urbanum. Evolutionary Ecology 24 (6): 1353-1364.

Willi, Y., Fischer, M. 2005. Genetic rescue in interconnected populations of small and large size of self-incompatible Ranunculus reptans. Heredity 95(6): 437-443.

WILLIS, J.H. 1992. Genetic analysis of inbreeding depression caused by chlorophyll-deficient lethals in Mimulus guttatus. Heredity 69: 562-572.

Recibido: 28.11.2016

Aceptado: 25.09.2017 